\title{
Aumento de resistencia de Streptococcus agalactiae vaginal-anal en el tercer trimestre de gestación a eritromicina y clindamicina al cabo de una década de tamizaje universal
}

\author{
Fernando Abarzúa C., Alejandra Arias E., Patricia García C., \\ Constanza Ralph T., Jaime Cerda L., Ingrid Riedel K. y Cynthia Gárate O.
}

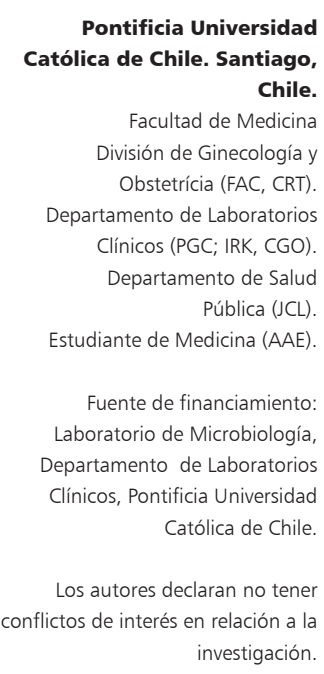

Recibido: 24 de agosto de 2010 Aceptado: 23 de mayo de 2011

Correspondencia a: Fernando Abarzúa Camus fabarzua@med.puc.cl.

\section{Streptococcus agalactiae increase in resistance to erythromycin and clindamycin in vaginal-anal colonization in third quarter of pregnancy in one decade of universal screening}

Introduction: Streptococcus agalactiae (GBS) is the main causative agent of early perinatal sepsis. The acquisition of prevention policies has led to frequent use of intrapartum antibiotics. Surveillance of antimicrobial resistance is indispensable for defining drugs of choice and alternatives for such prophylaxis. Objectives: To determine the evolution of antimicrobial resistance of GBS from maternal colonization to drugs used in the prevention of neonatal sepsis, between 2002 and 2008. Methods: We studied 100 GBS positive vaginal and anal samples from pregnant women. Disc diffussion susceptibility method was performed for penicillin, ampicillin, cefazolin, erythromycin and clindamycin according to the Clinical and Laboratory Standards Institute (CLSI). Results: We analyzed the susceptibility of 99 strains. Seventeen were resistant to erythromycin $(17.1 \%)$ and 13 were resistant to clindamycin (13.1\%). Thirteen of the 17 strains resistant to erythromycin had the MLS phenotype (resistance to erythromycin and clindamycin) and 4 had the M phenotype (resistance to erythromycin only). Within the MLS phenotype, resistance was constitutive in 9 strains, and induced in 4 strains (positive D test). Compared with 2002 there was a significant increase in resistance to clindamycin (from $3.27 \%$ to $13.1 \% \mathrm{p}<0.002$ ) and erythromycin $(1.09 \%$ to $17 \%$ p < 0.001$) .100 \%$ GSB remained sensitive to penicillin and ampicillin. Conclusions: GBS remains highly susceptible to drugs of choice for prevention of perinatal sepsis. There is a significant increase in antimicrobial resistance to clindamycin and erythromycin. Therefore, it is necessary to request susceptibility testing in GBS from third trimester of pregnancy screening in patients allergic to penicillin.

Key words: Streptococcus agalactiae (group B), antimicrobial susceptibility, neonatal sepsis, pregnancy.

Palabras clave: Streptococcus agalactiae (grupo B), susceptibilidad antimicrobiana, sepsis neonatal, embarazo.

\section{Introducción}

treptococcus agalactiae (Streptococcus $\beta$-hemolítico grupo B) es el principal agente causal de sepsis perinatal precoz, con una incidencia de $2-3 / 1.000$ partos y una mortalidad establecida en $15 \%{ }^{1}$. La adquisición de políticas de prevención ha traído consigo la utilización frecuente de antimicrobianos intraparto logrando una reducción significativa de casos en hijos de pacientes colonizadas con S. agalactiae.

Existen dos aproximaciones para un uso adecuado de profilaxis antimicrobiana: el protocolo de tamizaje que ha logrado pesquisar a $20 \%$ de aquellas mujeres en gestación y colonizadas realizando cultivo vaginal-anal entre las $35^{\circ}$ y $37^{\circ}$ semanas del embarazo; por otra parte, el protocolo de factores de riesgo ha logrado pesquisar a 10 a $15 \%$ de mujeres embarazadas, considerando a la fiebre intraparto, prematurez, rotura prematura de membranas (RPM) prolongada y a la historia previa de infección neonatal por S. agalactiae como condiciones que aumentan el riesgo ${ }^{2}$.

Existen esquemas profilácticos de primera línea con penicilina y ampicilina, además de fármacos de segunda línea indicados en pacientes alérgicas a $\beta$-lactámicos, con eritromicina o clindamicina. La vigilancia de resistencia antimicrobiana se hace indispensable para definir los fármacos de elección y alternativas en dicha profilaxis.

Nuestro centro realiza un tamizaje universal y en el año 2002 publicó sus patrones de resistencia ${ }^{3}$. Históricamente resultó positivo el cultivo en $20 \%$ de las mujeres embarazadas en nuestro hospital.

Los objetivos de este estudio son determinar la resistencia antimicrobiana de $S$. agalactiae de colonización 
materna en el tercer trimestre de gestación, en el año 2008, a los antimicrobianos utilizados como elección o alternativas en la prevención de sepsis neonatal y comparar el patrón de resistencia con lo reportado por nuestro centro en el año 2002.

\section{Pacientes y Métodos}

El Hospital Clínico de la Pontificia Universidad Católica de Chile realiza desde hace más de una década tamizaje universal de portación de $S$. agalactiae a las mujeres embarazadas en el tercer trimestre de gestación, según protocolo publicado (Abarzúa, Guzmán y cols 2002) $)^{3 a, 4}$. Dicho protocolo de manejo clínico se mantiene inalterable hasta el día de hoy.

Durante el año 2008 se estudiaron 100 muestras vaginales-anales consecutivas de mujeres embarazadas con edad gestacional de 35 a 37 semanas, que resultaron positivas para S. agalactiae. Las muestras fueron sembradas en caldo de cultivo Todd Hewitt (BBL $\AA$, Beckton Dickinson) suplementado con gentamicina $(8 \mu \mathrm{g} / \mathrm{ml})$ y ácido nalidixico $(15 \mu \mathrm{g} / \mathrm{ml})$, y posteriormente resembradas en agar sangre. A las colonias $\beta$-hemolíticas recuperadas se les realizó test de CAMP y/o aglutinación por látex para S. agalactiae (Streptex ${ }^{\circledR}$, Murex Diagnostic INC). Todas las cepas aisladas fueron sometidas a estudio de sensibilidad por difusión en agar para penicilina, ampicilina, cefazolina, clindamicina y eritromicina, según método estandarizado por Clinical and Laboratory Standards Institute (CLSI) ${ }^{4,5}$.

El test estadístico utilizado fue $\chi^{2}$, con un nivel de significancia de $\mathrm{p}<0,05$ considerado como estadísticamente significativo.

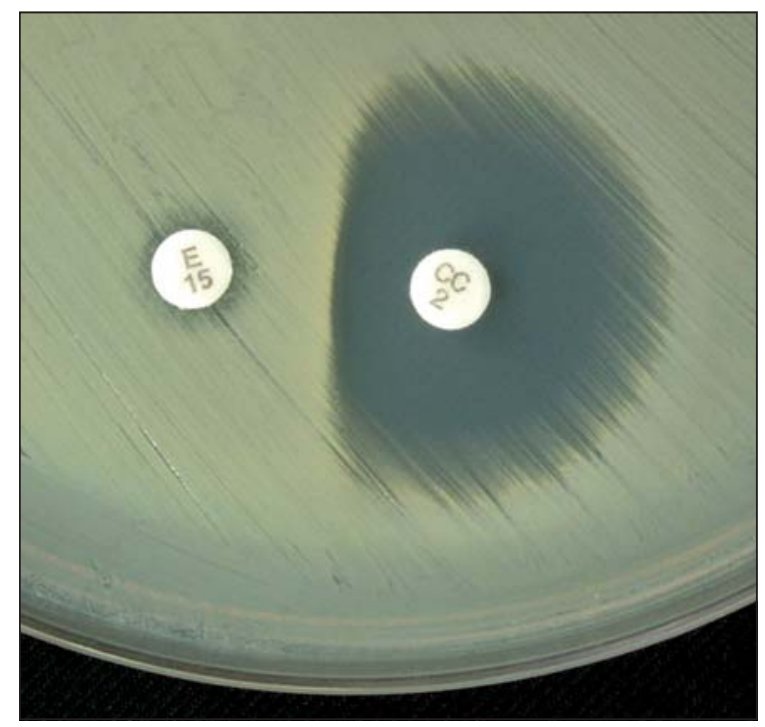

Figura 1. Test $D$.

\section{Resultados}

De las cepas estudiadas, en 99 se evaluó la susceptibilidad in vitro. De éstas, 17 (17,1\%) fueron resistentes a eritromicina y 13 resistentes a clindamicina $(13,1 \%)$. De las 17 cepas resistentes a eritromicina, 13 eran del fenotipo MLS. Cuatro fueron de fenotipo M. Dentro del fenotipo MLS, la resistencia fue constitutiva en nueve cepas, e inducible en cuatro cepas (Figura 1). Para el análisis, una cepa intermedia se consideró como no susceptible. Comparado con el año 2002, hubo un aumento significativo de resistencia a clindamicina (de 3,27\% a 13,1\% p < 0,002) y a eritromicina (de 1,09\% a 17\% p < 0,001) (Figura 2). Streptococcus agalactiae se mantuvo $100 \%$ sensible a penicilina y ampicilina.

\section{Discusión}

El trabajo de Belmar y cols ${ }^{3}$, realizado en nuestro centro en 2002, sobre el análisis de 183 cepas, estableció $100 \%$ de sensibilidad a penicilina, ampicilina, cefazolina y vancomicina con una discreta resistencia a clindamicina y eritromicina de 3,3 y 1,1\%, respectivamente ${ }^{3}$. En esta nueva evaluación, hemos observado un aumento significativo de resistencia para los dos últimos antimicrobianos mencionados: 13 y 17\% respectivamente. Trece eran del fenotipo MLS -explicado por metilación de la subunidad 23s ribosomal- lo que confiere resistencia a eritromicina y clindamicina y cuatro fueron de fenotipo $\mathrm{M}$-explicado

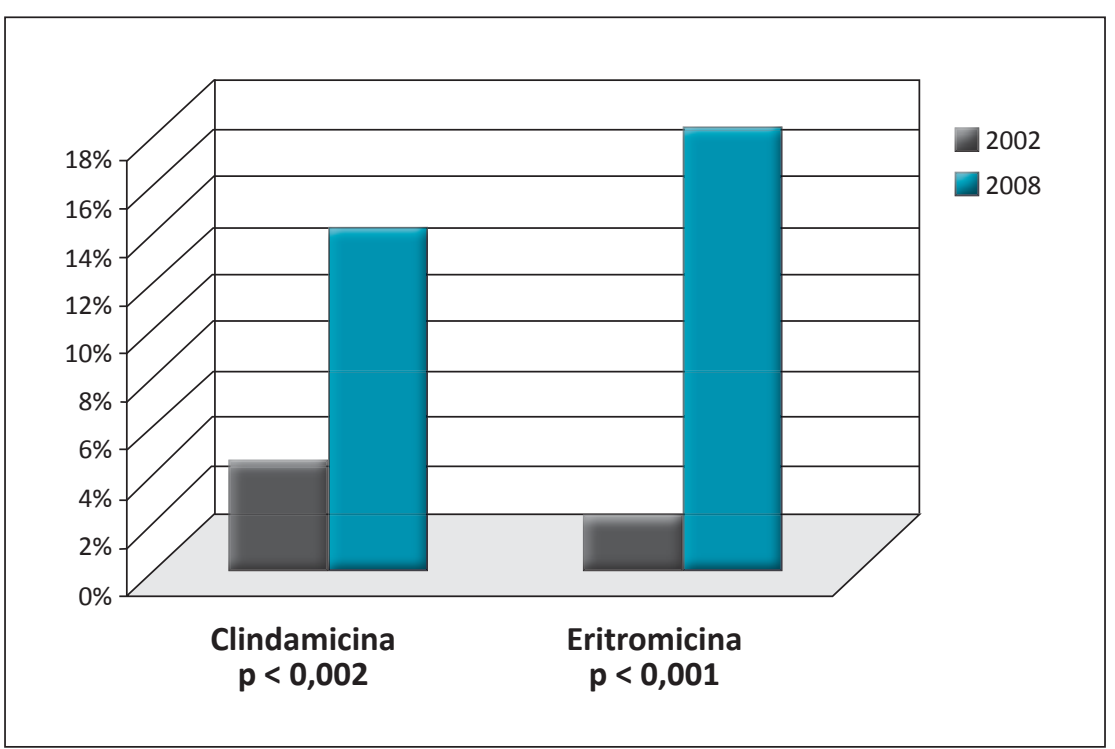

Figura 2. Resistencia antimicrobiana de cepas de Streptococcus agalactiae de colonización vaginalanal en mujeres embarazadas de tercer trimestre. Hospital Clínico Pontificia Universidad Católica de Chile. Comparación de períodos 2002 y 2008. 
por bombas de expulsión-el que confiere sólo resistencia a eritromicina.

Este aumento de resistencia es considerable y llamativo dado el uso mínimo de estos antimicrobianos para la profilaxis de infecciones causadas por S. agalactiae, suponiendo que aproximadamente $10 \%$ de la población presenta una respuesta alérgica leve a $\beta$-lactámicos y una cifra menor aún de anafilaxis (entre 4/10.000 y 4/100.000 casos). En la gran mayoría de las mujeres embarazadas que reciben profilaxis intraparto, se utiliza penicilina o ampicilina, antimicrobianos que han mantenido $100 \%$ de actividad sobre S. agalactiae. La resistencia ha surgido, entonces, en fármacos que se indican muy infrecuentemente para estos fines.

Es altamente probable que la resistencia haya aumentado por la "presión ecológica" que ejerce el uso de clindamicina y eritromicina en otras patologías médicas, mencionando como ejemplo el amplio uso de macrólidos en patología respiratoria o, en obstetricia, el manejo de corioamnionitis durante la gestación y endometritis en el puerperio ${ }^{5,6}$.

Cada vez más centros en diversas partes del mundo han incorporado el tamizaje universal con cultivo al término del embarazo para reducir la infección perinatal por $S$. agalactiae. Por ello, se dispone de información acerca de la susceptibilidad antimicrobiana de esta bacteria (Tabla 1). Spatgens y cols, realizaron una revisión en 235 mujeres embarazadas con 36 semanas de gestación, evidenciando 3 y $5,6 \%$ de resistencia a clindamicina y eritromicina, respectivamente $^{6,7}$. Al-Sweih y cols (año 2003), describieron un perfil de susceptibilidad antimicrobiana en un total de 1.166 muestras vaginales-rectales obteniendo $1,7 \%$

\begin{tabular}{|c|c|c|c|c|}
\hline \multirow[t]{2}{*}{ Estudio } & \multirow[t]{2}{*}{ Año } & \multicolumn{2}{|c|}{ Frecuencia de resistencia (\%) } & \multirow[t]{2}{*}{$\mathbf{n}$} \\
\hline & & Clindamicina & Eritromicina & \\
\hline Abarzúa F. - Chile & 2011 & 13,10 & 17,10 & 99 \\
\hline Garland SM., Australia & 2011 & 4,20 & $6,40 \%$ & 1.160 \\
\hline Dhanoa A., Malasia & 2010 & 2,50 & 4,00 & 200 \\
\hline Joachim A., Tanzania & 2009 & 17,60 & 13,00 & 69 \\
\hline Shabayek SA., Egipto & 2009 & 13,15 & 23,68 & 150 \\
\hline Hsiu-Mei Wu, Taiwan & 2008 & 62,00 & 65,00 & 1994 \\
\hline Gygax SE., E.U.A. & 2006 & 21,0 & 38,00 & 222 \\
\hline González JJ, España & 2004 & 11,80 & 12,50 & 610 \\
\hline José A. Simoes, E.U.A. & 2004 & 19,00 & - & 52 \\
\hline N. Al-Sweih, Kuwait & 2005 & 1,70 & 0,70 & 1166 \\
\hline Belmar C. - Chile & 2002 & 3,30 & 1,10 & 183 \\
\hline Spaetgens R., Canadá & 2002 & 3,00 & 5,60 & 235 \\
\hline
\end{tabular}

de resistencia a clindamicina y $0,7 \%$ a eritromicina ${ }^{7,8}$. Simoes y cols, en el 2004 determinaron la resistencia de $S$. agalactiae a 12 antimicrobianos en 52 mujeres no embarazadas y recién nacidos; fue posible encontrar resistencia a clindamicina de $19,2 \%$ y eritromicina $25 \%$, sin resistencia a penicilina ni ampicilina pero sí con susceptibilidades intermedias de 15,4 y 17,3\%, respectivamente ${ }^{8,9}$. González y cols, en el 2004, en un estudio multicéntrico, evaluaron la susceptibilidad in vitro de 610 cepas de S. agalactiae, extraídas principalmente de mujeres embarazadas con 35 a 37 semanas de gestación, y un número mínimo de LCR de neonatos menores de 7 días. La resistencia a clindamicina fue de $11,8 \%$ y a eritromicina de $12,5 \% 0^{9,10}$. En 2006, Gygax y cols, evaluaron la susceptibilidad in vitro de 222 cepas de $S$. agalactiae con determinación de genes de resistencia. Obtuvo resistencias para clindamicina y eritromicina de 21 y $38 \%$, respectivamente ${ }^{10,11}$. En un estudio más reciente (año 2008), Hsiu-Mei Wu y $\operatorname{cols}^{11,12}$, encontraron un aumento importante de resistencia a clindamicina y eritromicina: 62 y $65 \%$, respectivamente.

En los últimos tres años, regiones tan diferentes como Australia y Malasia muestran resistencias relativamente bajas, tanto a clindamicina ( 4,2 y $2,5 \%)$ como a eritromicina (6,4 y 4\%); en cambio, desde Egipto y Tanzania se han comunicado resistencias más elevadas, y que están más en concordancia con lo que sucede en nuestra experiencia ${ }^{13-16}$.

Un autor australiano, Garland SM y cols, (año 2011), hizo un análisis de un posible cambio de resistencia entre dos períodos en un total de 1.160 muestras, tal como reportamos nosotros en este artículo. A diferencia de nuestros resultados, ellos no encontraron modificaciones en el patrón de susceptibilidad a macrólidos entre los períodos 1982-2001 y 2002-2006 ${ }^{13}$.

Cabe destacar que todos estos estudios, salvo uno, coinciden en que aún no existe resistencia a penicilina ni ampicilina. Sólo la experiencia de Tanzania ${ }^{15}$, reporta una resistencia de $9,4 \%$ a penicilina, manteniendo $100 \%$ de susceptibilidad a ampicilina. Por ello, ambos antimicrobianos siguen siendo de elección para profilaxis intraparto.

La frecuencia de resistencia antimicrobiana debe ser vigilada en cada centro asistencial. Es importante destacar que en el nuestro, la resistencia a clindamicina y eritromicina ha aumentado significativamente, como lo han reportado algunos otros autores, lo que nos obliga a tener un manejo más responsable y adecuado en el uso de antimicrobianos. Este incremento de resistencia aumenta el riesgo de hijos de madres alérgicas a $\beta$-lactámicos, de adquirir una infección por S. agalactiae. Además se vislumbra el eventual fracaso terapéutico en otras patologías del embarazo que son tratadas con estos antimicrobianos, aumentando también los riesgos de morbi-mortalidad neonatal.

Para aminorar esta problemática actual se hace necesario (junto al uso racional de antimicrobianos), solicitar 
antibiograma en el tamizaje del tercer trimestre del embarazo, en aquellas pacientes con el antecedente de alergia a penicilina, con el fin de disminuir y/o detener el aumento de resistencia y dar un tratamiento efectivo a estas madres. Sin duda, es fundamental para cada institución de salud actualizar con frecuencia sus patrones de susceptibilidad antimicrobiana.

\section{Resumen}

Introducción: Streptococcus agalactiae es el principal agente causal de sepsis perinatal precoz. La adquisición de políticas de prevención ha traído consigo la utilización frecuente de antimicrobianos intra-parto. La vigilancia de resistencia antimicrobiana se hace indispensable para definir el fármaco de elección y alternativas en dicha profilaxis. Nuestro centro realiza tamizaje universal desde hace 10 años. Objetivos: Determinar la evolución de la resistencia antimicrobiana de S. agalactiae de colonización materna, a los antimicrobianos utilizados en la prevención de sepsis neonatal, entre 2002 y 2008. Métodos: Se estudiaron 100 muestras vaginales-anales positivas para $S$. agalactiae de mujeres embarazadas, con edad gestacional de 35 a 37 semanas. Se realizó estudio de susceptibilidad in vitro por discos a penicilina, ampicilina, cefazolina, eritromicina y clindamicina, según método estandarizado por Clinical and Laboratory Standards Institute (CLSI). Resultados: Se analiza la susceptibilidad de 99 cepas. Diecisiete fueron resistentes a eritromicina $(17,1 \%)$ y 13 eran resistentes a clindamicina $(13,1 \%)$. De las 17 cepas resistentes a eritromicina, 13 eran fenotipo MLS y 4 del fenotipo M. Dentro del fenotipo MLS, la resistencia fue constitutiva en nueve cepas e inducible en cuatro cepas (test D positivo). En comparación con el año 2002, hubo un aumento significativo de resistencia a clindamicina (de 3,2 a $13,1 \%$ p < 0,002) y a eritromicina (de 1,09 a $17 \% \mathrm{p}<0,001)$. Streptococcus agalactiae se mantuvo $100 \%$ sensible a penicilina y ampicilina. Conclusiones: S. agalactiae mantiene alta sensibilidad a los antimicrobianos de elección para la prevención de sepsis neonatal y a un antimicrobiano alternativo: cefazolina. Se observó un aumento significativo de resistencia antimicrobiana a clindamicina y eritromicina. Se hace necesario, entonces, solicitar antibiograma en el tamizaje del tercer trimestre del embarazo, en pacientes alérgicas a penicilina.

\section{Referencias}

1.- Cruz O M, Doren V A, Tapia I JL, Abarzúa C F. Sepsis neonatal por Streptococcus grupo B. Rev Chil Pediatr 2008; 79 (5): 462-70.

2.- Schrag S, Gorwitz R, Fultz-Butts K, Schuchat A. Prevention of perinatal group B streptococcal disease. Revised guidelines from CDC. MMWR Morb Mortal Wkly Rep 2002; 51 RR.11: 1-22.

3.- Belmar J C, Abarzúa C F, Becker V J, Guzmán D AM, García C P, Oyarzún E E. Estudio de sensibilidad antimicrobiana de 183 cepas de Streptococcus agalactiae aisladas en región vagino-perineal de embarazadas en el tercer trimestre. Rev Chil Obstet Ginecol 2002; 67 (2): 106-9.

4.- Abarzúa C F, Guzmán D AM, Belmar J C, Becker V J, García C P, Rioseco A, et al. Prevalencia de colonización por Streptococcus agalactiae (grupo B) en el tercer trimestre del embarazo. Evaluación del cultivo selectivo. Experiencia en 2.192 pacientes. Rev Chil Obstet Ginecol 2002; 67 (2): 89-93.

5.- Clinical and Laboratory Standars Institute. Performance Standards for Antimicrobial Susceptibility Testing 2010. M100 - S20. Vol. 30 No. 1.

6.- Oyarzún E. Rotura prematura de membranas, Oyarzún E E, Badía A J, Alto Riesgo Obstétrico,
Primera edición. Santiago de Chile. Ediciones Universitarias PUC, 1997; p. 97-112.

7.- $\quad$ Spaetgens R, DeBella K, Ma D, Robertson S, Mucenski M, Davies H D. Perinatal antibiotic usage and changes in colonization and resistance rates of group B Streptococcus and other pathogens. Obstet Gynecol 2002; 100: 525-33.

8.- Al-Sweih N, Jamal M, Kurdia M, Abduljabar R, Rotimi V. Antibiotic susceptibility profile of group B Streptococcus (Streptococcus agalactiae) at the Maternity Hospital, Kuwait. Med Princ Pract 2005;14 (4): 260-3.

9.- Simoes JA, Aroutcheva AA, Heimler I, Faro S. Antibiotic resistance patterns of group B streptococcal clinical isolates. Infect Dis Obstet Gynecol 2004; 12: 1-8.

10.- González J J, Andreu A; Grupo de Estudio de Infección Perinatal, Sociedad Española de Enfermedades Infecciosas y Microbiología Clínica. Susceptibility of vertically transmitted group B streptococci to antimicrobial agents. Enferm Infecc Microbiol Clin 2004; 22 : 286-91.

11.- Gygax S E, Schuyler J A, Kimmel L E, Trama J P, Mordechai E, Adelson M E. Erythromycin and clindamycin resistance in group B streptococcal clinical isolates. Antimicrob Agents Chemother 2006; 50: 1875-7.
12.- Wu H M, Janapatla R P, Ho Y R, Hung K H, Wu C W, Yan J J, et al. Emergence of fluoroquinolone resistance in group B streptococcal isolates in Taiwan. Antimicrob Agents Chemother 2008; 52: 1888-90.

13.- Garland S M, Cottrill E, Markowski L, Pearce C, Clifford V, Kelly N, et al, Australasian Group for Antimicrobial Resistance-GBS resistance Group. Antimicrobial resistance in group B streptococcus: the Australian experience. J Med Microbiol 2011; 60 (Pt 2): 230-5.

14.- Dhanoa A, Karunakaran R, Puthucheary S D. Serotype distribution and antibiotic susceptibility of group B streptococci in pregnant women. Epidemiol Infect 2010; 138 (7): 979-81.

15.- Joachim A, Matee M I, Massawe F A, Lyamuya E F. Maternal and neonatal colonization of group B streptococcus at Muhimbili National Hospital in Dar es Salaam, Tanzania: prevalence, risk factors and antimicrobial resistance. BMC Public Health 2009; 9: 437.

16.- Shabayek S A, Abdalla S M, Abouzeid A M. Vaginal carriage and antibiotic susceptibility profile of group B streptococcus during late pregnancy in Ismailia, Egypt. J Infect Public Health 2009; 2 (2): 86-90. 